\title{
A REMARK ON THE RADICAL OF A GROUP ALGEBRA
}

\author{
by PETER BROCKHAUS
}

(Received 5th July 1980)

This paper contains a new proof of a theorem of D. A. R. Wallace [5] in case of a $p$-solvable group. An alternative proof has been given by $\mathrm{K}$. Motose [4].

Let $G$ be a finite group, $F$ a field with prime characteristic $p, P$ a Sylow $p$-subgroup of $G$. JFG will denote the Jacobson radical of $F G$.

Lemma 1. Let $Q$ be a p-group acting on a $p^{\prime}$-group $H$ such that $Q$ stabilises each conjugacy class of $H$. Then $Q$ acts trivially.

Proof. Let $K$ be a conjugacy class of $H$. By assumption $Q$ acts on $K$. Since $p \nmid|K|$ there exists $k \in K$ such that $k \in C_{H}(Q)$ implies

$$
K=k^{H} \subseteq \bigcup_{h \in H}\left[C_{H}(Q)\right]^{h}
$$

It follows $H=\bigcup_{h \in H}\left[C_{H}(Q)\right]^{h}$. By [2], p. 12, Aufgabe 4, $H=C_{H}(Q)$.

Lemma 2. Assume $G=P N$ where $N:=O_{p^{\prime}}(G)$. Then

$$
\operatorname{dim}_{F} J F G=|G|-|N| \Rightarrow p \unlhd G .
$$

Proof. Since $F N \cap J F G \subseteq J F N=0$ (by Maschke's theorem) the map

$$
\varphi: F N \rightarrow F G / J F G \quad \alpha \mapsto \alpha+J F G
$$

is an $F$-algebra monomorphism. $\varphi$ is an isomorphism, since $\operatorname{dim}_{F}(F G / J F G)=$ $|G|-\operatorname{dim}_{F} J F G=|N|$. Define $\Psi: F G \rightarrow F N$ by $\Psi(\alpha):=\varphi^{-1}(\alpha+J F G)(\alpha \in F G) . \Psi$ is an $F$-algebra homomorphism with property $\left.\Psi\right|_{F N}=1_{F N}$. Let $K$ be a conjugacy class of $N$ and $x \in P$.

$$
c:=\sum_{k \in K} k \in Z F N
$$

$c^{x}=\Psi\left(c^{x}\right)=\Psi\left(x^{-1}\right) \Psi(c) \Psi(x)=c^{u}=c$, where $u:=\Psi(x)$, a unit in $F N \Rightarrow K^{x}=K \Rightarrow P$ acts on $K$ by conjugation. By Lemma $1 N=C_{N}(P)$. 


\section{Lemma 3.}

(a) $H \leqq G \Rightarrow \operatorname{dim}_{F} J F G \leqq \operatorname{dim}_{F} J F H+|G|-|H|$;

(b) $\operatorname{dim}_{F} J F G \leqq|G|-|G: P|$, if $G$ has a p-complement;

(c) $H \unlhd G, p \nmid|G: H| \Rightarrow \operatorname{dim}_{F} J F G=|G: H| \operatorname{dim}_{F} J F H$;

(d) $Q \unlhd G, Q$ a p-group $\Rightarrow \operatorname{dim}_{F} J F G=\operatorname{dim}_{F} J F(G / Q)+|G|-|G: Q|$.

Proof. (a) $x+J F G \cap F H \mapsto x+F H$ for all $x \in J F G$ defines an $F$-monomorphism from the $F$-vector space $J F G / J F G \cap F H$ into the $F$-vector space $F G / F H$. Therefore, since $J F G \cap F H \subseteq J F H$,

$$
\begin{aligned}
\operatorname{dim}_{F} J F G & =\operatorname{dim}_{F}(J F G / J F G \cap F H)+\operatorname{dim}_{F}(J F G \cap F H) \\
& \leqq \operatorname{dim}_{F}(F G / F H)+\operatorname{dim}_{F} J F H=|G|-|H|+\operatorname{dim}_{F} J F H .
\end{aligned}
$$

(b) Let $H$ be a $p$-complement. Apply (a) and Maschke's theorem.

(c) follows from [3], p. 524.

(d) follows easily from [1], p. 71-72.

Theorem (Wallace [5]). Let $G$ be p-solvable. Then

$$
P \unlhd G \Leftrightarrow \operatorname{dim}_{F} J F G=|G|-|G: P| .
$$

Proof. " $\Rightarrow$ ":

$$
\begin{aligned}
\operatorname{dim}_{F} J F G & =\operatorname{dim}_{F} J F(G / P)+|G|-|G: P| \quad \text { (by Lemma 3(d)) } \\
& =|G|-|G: P| \quad \text { (by Maschke's theorem) }
\end{aligned}
$$

" $\Leftarrow$ ": We use induction on $|G|$. The case $|G|=1$ is clear. Assume that $\| G \mid>1$ and the result holds for $p$-solvable groups of smaller order. As $G$ is $p$-solvable, there exists $H \nsubseteq G: p \nmid|G: H|$ or $|G: H|=p$.

Case 1: $p \nmid|G: H|$. Then $P \in \operatorname{Syl}_{p}(H)$.

$$
\begin{aligned}
\operatorname{dim}_{\mathrm{F}} J F H & =|G: H|^{-1} \operatorname{dim}_{F} J F G \quad(\text { by Lemma 3(c)) } \\
& =|G: H|^{-1}(|G|-|G: P|)=|H|-|H: P|
\end{aligned}
$$

$\Rightarrow P \unlhd H$ (by induction) $\Rightarrow P \unlhd G \quad$ (since $P$ char $H \unlhd G)$.

Case 2: $|G: H|=p$.

Case 2a: $\quad p \nmid|H|$. Then $H=O_{p},(G)$ and, by Lemma 2, $P \leq G$.

Case 2b: $p|| H \mid$. Let $Q \in \operatorname{Syl}_{p}(H)$.

$$
\begin{aligned}
& \operatorname{dim}_{F} J F H \geqq \operatorname{dim}_{F} J F G+|H|-|G| \quad(\text { by Lemma 3(a)) } \\
&=|G|-|G: P|+|H|-|G|=|H|-|G: P|=|H|-|H: Q| \\
& \Rightarrow \operatorname{dim}_{F} J F H=|H|-|H: Q| \quad(\text { by Lemma 3(b)) } \\
& \Rightarrow Q \unlhd H(\text { by induction) } \Rightarrow Q \unlhd G \quad(\text { since } Q \text { char } H \unlhd G) \\
& \Rightarrow \operatorname{dim}_{F} J F(G / Q)=\operatorname{dim}_{F} J F G+|G: Q|-|G| \quad(\text { by Lemma 3(d)) } \\
&=|G|-|G: P|+|G: Q|-|G| \\
&=|G / Q|-|G / Q: P / Q|
\end{aligned}
$$

By induction $P / Q \unlhd G / Q$ (since $P / Q \in \operatorname{Syl}_{p}(G / Q)$ ) and therefore $P \unlhd G$. 
In conclusion I wish to thank Professor G. Michler for helpful discussions, Dr. M. Lorenz for improvements of the original version and the referee for a useful hint.

\section{REFERENCES}

1. W. Hamernik. Group Algebras of Finite Groups Lectures given at the University of Giessen, Winter 1973/74.

2. B. HuPpert, Endliche Gruppen I (Springer, Berlin, Heidelberg, New York, 1967).

3. G. Michler, Blocks and Centers of Group Algebras (Lecture Notes in Mathematics 246, Springer, 1972).

4. K. Motose, On a Theorem of Wallace and Tsushima, Proc. Japan Acad. 50 (1974), $572-575$.

5. D. A. R. Wallace, On the Radical of a Group Algebra, Proc. Amer. Math. Soc. 12 (1961), 133-137.

Department of Mathematics

ESSEN UNIVERSITY

4300 ESSEN

Federal Republic of Germany 